\title{
Electron Tomography and Synapse Study
}

\author{
Hyun-Wook Kim, Dasom Kim, Im Joo Rhyu*
}

Department of Anatomy, Korea University College of Medicine, Seoul 136-705, Korea

*Correspondence to:

Rhyu IJ,

Tel: $+82-2-2286-1149$

Fax: +82-2-929-5969

E-mail: irhyu@korea.ac.kr

Received September 12, 2014

Revised September 23, 2014

Accepted September 23, 2014

\begin{abstract}
Electron tomography (ET) is a useful tool to investigate three-dimensional details based on virtual slices of relative thick specimen, and it requires complicated procedures consisted of image acquisition steps and image processing steps with computer program. Although the complicated step, this technique allows us to overcome some limitations of conventional transmission electron microscopy: (1) overlapping of information in the ultrathin section covering from $30 \mathrm{~nm}$ to $90 \mathrm{~nm}$ when we observe very small structures, (2) fragmentation of the information when we study larger structures over $100 \mathrm{~nm}$. There are remarkable biological findings with ET, especially in the field of neuroscience, although it is not popular yet. Understanding of behavior of synaptic vesicle, active zone, pooling and fusion in the presynaptic terminal have been enhanced thanks to ET. Some sophisticated models of postsynaptic density with ET and immune labeling are introduced recently. In this review, we introduce principles, practical steps of ET and some recent researches in synapse biology.
\end{abstract}

Key Words: Synapse, Neuroscience, Synaptic vesicle, Pooling, Postsynaptic density

\section{ELECTRON TOMOGRAPHY}

Electron tomography (ET) is a useful tool to investigate threedimensional (3D) details based on virtual slices of relative thick specimen. A single electron transmission microscopic image is to ET what single X-ray image is to computed X-ray tomography (Rhyu \& Park, 2008). As computed X-ray tomographic image shows cross sectional information of the body without real sectioning in hospital, we can get virtual cross sectional image of relative thick specimen up to resolution $2.4 \AA$ recently (Scout et al., 2012). Not only technical upgrade of ET, but sophisticated analyses of various specimens are main interest of electron tomographic studies (Frey et al., 2006; Mun et al., 2008; Jou et al., 2013). By using relative thick specimen and very thin virtual slice up to 1 $\mathrm{nm}$, ET is a useful tool dissecting very small structures and relatively large structures at the same time.

\section{PRINCIPLES OF ELECTRON TOMOGRAPHY}

Transmission electron microscopes (TEM) typically get projection images. Thus the image contains information from all levels of the specimen flattened onto a single plane. If we take images in tilting angle by regular step of degree, the information can be extracted as we can catch 3D information from stereo paired images of TEM or high voltage electron microscopy (Lee et al., 2004). But, the 3D information from stereo pair images depends on merging the image at the brain and two and half dimension (Kim et al., 2007). Therefore, there is some limitation understanding real 3D structure of the specimen.

The more exact 3D information would be available if we add more tilt series image. This can be achieved by "back projection procedures" based on various algorithms, which let us manage virtual specimen block. We can manipulate this virtual specimen block for further analysis (Fig. 1). The quality and resolution are determined by range of tilting steps, back projection algorithm.

This work was supported by Basic Science Research Program through the National Research Foundation (NRF-2013R1A1A2060344).

(a) This is an open-access article distributed under the terms of the Creative Commons Attribution Non-Commercial License (http://creativecommons.org/licenses/by-nc/3.0) which permits unrestricted noncommercial use, distribution, and reproduction in any medium, provided the original work is properly cited.

Copyrights $\odot 2014$ by Korean Society of Microscopy 
A

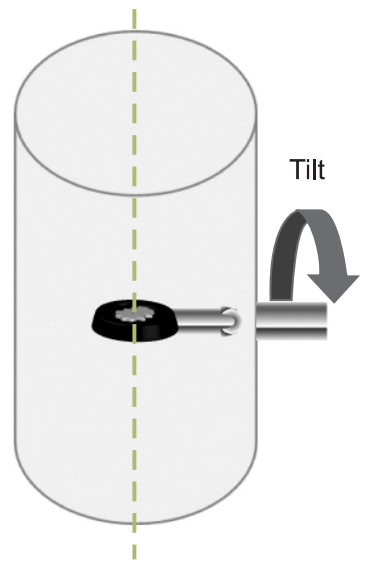

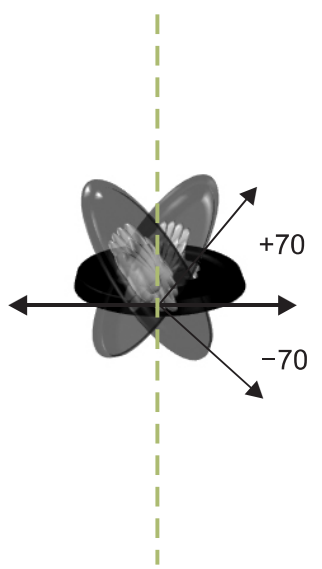

C

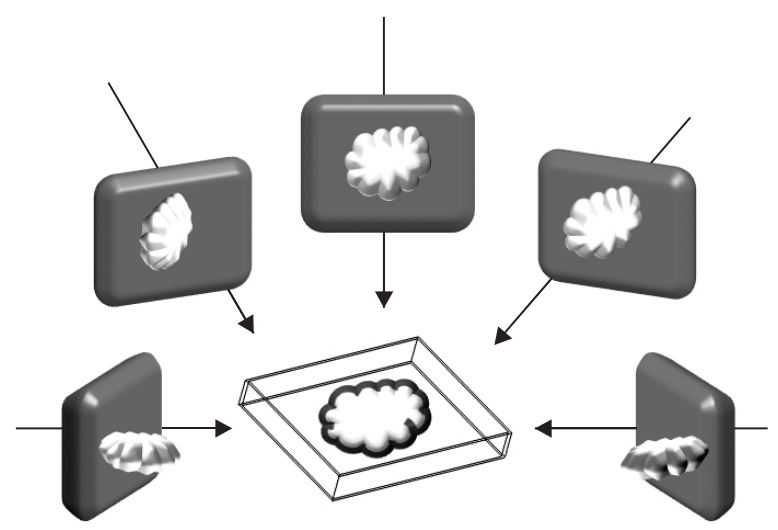

B

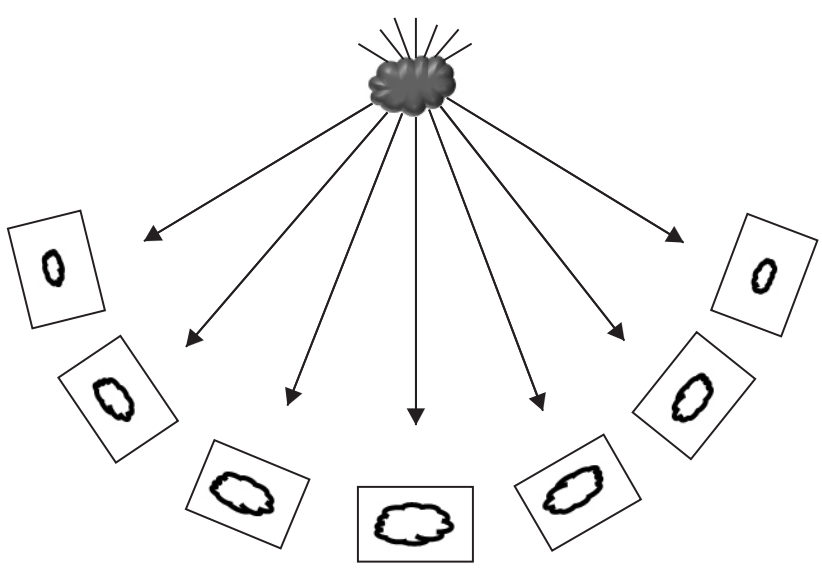

D

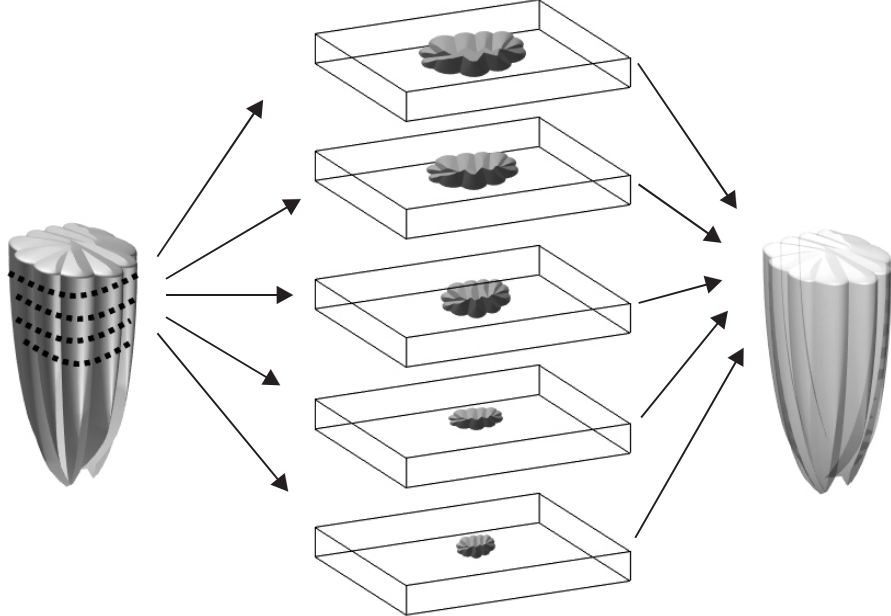

Fig. 1. Major steps of electron tomography. (A, B) Serial tiling images are captured by rotating holder and camera system. (C) Virtual block is constructed by computed back projection based on each tilted images. (D) The virtual block can be sliced according to research purpose and further imaging process.

\section{EXPERIMENTAL STEPS FOR ELECTRON TOMOGRAPHY}

The biological samples should be fixed for electron microscopic studies. Routine histologic preparation requires fixation of tissues. Chemical fixation is a long traditional method using chemicals such as formaldehyde, paraformaldelhyde, glutaraledhyde, aceton, alcohol and other chemicals, which are convenient to applicable in conventional laboratory. Some artificial and altered findings are reported in conventional routine chemical fixation when observed at ultrastructural level. ET with osmium free chemical fixation shows a new possibility to investigate synapse in detail (Burette et al., 2012). For efficient electron transmission, special plastic embedding resins are recommended.

Freezing fixation is supposed to preserve structure close to natural condition and useful to prevent from leakage

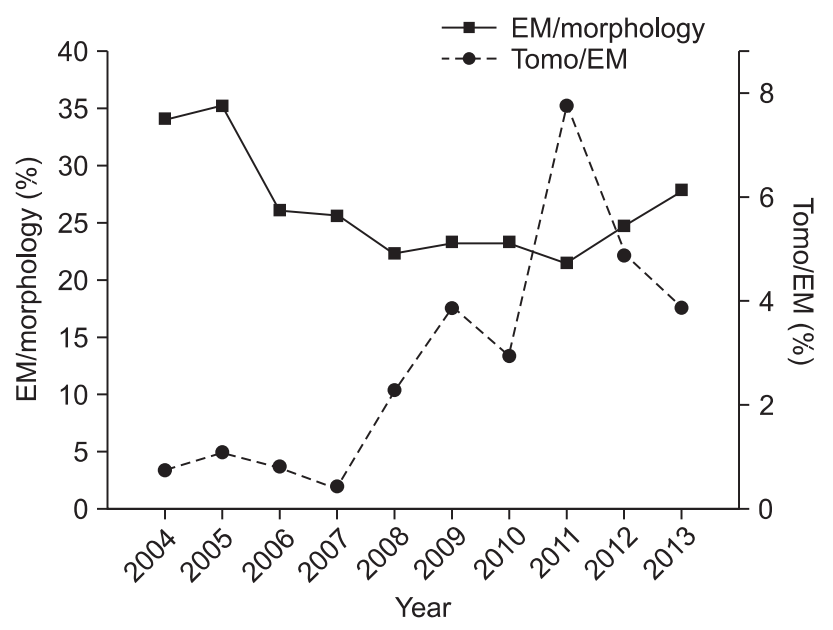

Fig. 2. Research trend on electron tomography (ET) based on PubMed database for recent 10 years. The conventional electron microscopy (EM) was employed in more than $26 \%$ of synapse researches and $2.9 \%$ of EM studies used ET. Recent increase of ET is remarkable. 
and kinking during chemical fixation. Analysis of abstracts presented at the 4 th international congress on ET shows that $44 \%$ of the study employed cryo-TEM (Rhyu \& Park, 2008). Recently the advantage of cryo-electron tomographic study has been introduced and employed in wide range of studies from single particle analysis to intracellular organelles
(McIntosh et al., 2005; Mun et al., 2008). Also recent progress in synapse biology presented in this review is also much debt on cryo-ET (Fernández-Busnadiego et al., 2011). The choice of fixation method is dependent on characteristics of specimen, purpose of research, and equipment of the laboratory.
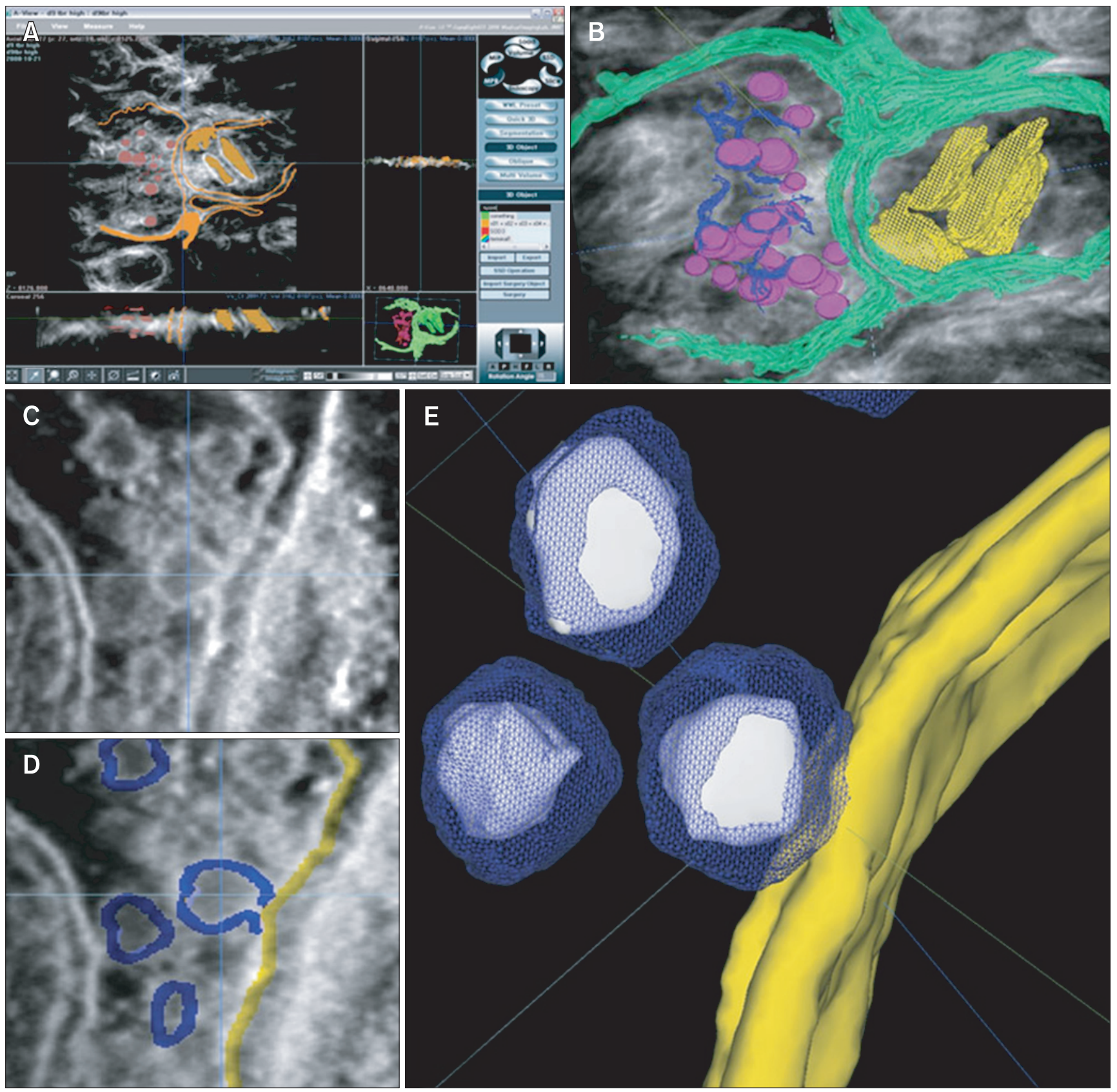

Fig. 3. Representative view of three-dimensional (3D) modeling synapse. (A) The editing status can be checked in three planes. (B) The fine filaments linking synaptic vesicles are clearly observed: purple, synaptic vesicles; blue, synaptic vesicle connection; green, pre- and postsynaptic membrane; yellow, endoplasmic reticulum in postsynaptic terminal. (C) The fusion of synaptic vesicle and presynaptic membrane shows typical omega-shaped morphology. (D, E) The 3D modeling of synaptic vesicle fused with presynaptic terminal is demonstrated after segmentation of synaptic vesicles and presynaptic membrane: blue, synaptic vesicle membrane; white, synaptic vesicle lumen; yellow, presynaptic membrane. Reused from the article of Kim et al. (2013; Microsc. Microanal. 19 Suppl 5, 182-187) with original copyright holder's permission. 
Once the samples are prepared, electron tomographic study follows 4 steps basically (Fig. 1): (1) acquisition of serially tilted images (i.e., a 'tilt-series'), (2) precise, digital alignment of adjacent images based on the trajectories of fiducial gold particles adhered to the sections before imaging begins, (3) computed reconstruction of an image volume from the aligned images to yield a 'virtual block,' typically using back-projection algorithms, and (4) generation and analysis of 1-pixel thick virtual image slices (Kim et al., 2013). These 4 series steps much depend on optic mechanics and computational techniques.

Electron microscopic systems for ET usually employed acceleration beam more than $200 \mathrm{kV}$. The specimen holder should be tilted by goniometer control and rotation of specimen holder is optional. A tilt series cab be taken by tilting the specimen holder regularly ( 1 or 2 degree step) about a fixed axis perpendicular to the electron beam and collecting images of the specimen ranged from -80 to +80 (Frey et al., 2006). The quality of final electron tomogram is dependent on increment step of tilting and overall tilting range. If double tilt series of image could be used for ET, missing wedge would be decreased and more exact tomography can be produced (Lučić et al., 2005). Recently some $100 \mathrm{kV}$ TEM models supporting electron tomographic function is commercially available (Kim et al., 2013).

Alignment and sewing of the tilted series images are a prerequisite steps for further ET processing. Alignment based on fiducial maker tracking using colloidal gold particle is widely adopted that each gold particle from each adjacent tilting series is marked and sewed. This method is exact and correct distorted image across the tilting series images, but requires a lot of input from researchers to finish the alignment, which is very tedious and time consuming step. Recently, automatic alignment systems free from fiducial marker are introduced by some major column company, which might be helpful for novice to practice ET studies (Kim et al., 2013). This system helps to save time, but still has weak point to correct distortion of tilting series during imaging and provide narrow area of ET.

After alignment, virtual block should be reconstructed, which is achieved by back projection step. Weighted back projection (WBP) is mostly adopted algorithm, because it provide fast reconstruction and is economic (freely available with IMOD [Colorado University, USA] package). In addition to this first line, some commercial algorithms such as simultaneous iterative reconstruction technique (SIRT), and topography based reconstruction (TBR) are available (Kim et al., 2013).

\section{INVESTIGATION OF SYNAPSE MORPHOLOGY USING ELECTRON TOMOGRAPHY}

Electron microscopy is a major tool studying morphology of synapse, since first confirmation of neuron doctrine suggested by Ramon y Cajal (Palade \& Palay, 1954). Pubmed data base search result based synapse morphology and electron microscopy for recent 10 years, showed that about $26 \%$ of researches studying synapse morphology employed electron microscopy and only $2.9 \%$ of the electron microscopic study for synapse used electron tomographic technique. However the recent increase of number of studies using ET is remarkable (Fig. 2).

The synapse is typically composed of presynaptic component, synaptic cleft, and postsynaptic component (Fig. 3). The ET has contributed remarkably to the advance of neuroscience research, especially synapse biology (Kim et al., 2013).

Several ET studies have characterized the morphometric features of synaptic structures at high resolution, such as number and size of synaptic vesicles, the area of presynaptic density and the architecture of the synaptic cleft distance (Siksou et al., 2007; Kim et al., 2013). The presynaptic terminals are filled with synaptic vesicles in filamentous matrix and mitochondria. The synaptic vesicles contain neurotransmitters, which are released into synaptic cleft. The role of filamentous structure was proposed by electron tomographic analysis. The long filamentous structures are identified as actin and short connectors (tethers) are suggested synapsin and additional components (Siksou et al., 2007). After stimulation of synapse, synaptic vesicles connected by tethers were decreased, compared to control experimental group (Fernández-Busnadiego et al., 2010). ET studies also suggested some models for docking mechanism and recycling of synaptic vesicles at the active zone (Landis et al., 1998; Siksou et al., 2007, 2009) and some molecular candidate for the synaptic vesicle pooling (FernándezBusnadiego et al., 2013). Studies on synaptic cleft are few, it is described as intercellular plaque similar to electron dense structure observed in dermal desmosomes (Lučić et al., 2007). The postsynaptic density is composed of very complicated components, although it looks like to be a just thickening of postsynaptic component under routine TEM. ET with immune-staining suggests illustration models in postsynaptic density proteins such as postsynaptic density protein 95 , $\mathrm{N}$-methyl-D-aspartate receptors, and $\alpha$-amino-3-hydroxy-5methyl-4-isoxazolepropionic acid receptor complex (Chen et al., 2008).

\section{CONFLICT OF INTEREST}

No potential conflict of interest relevant to this article was reported. 


\section{REFERENCES}

Burette A C, Lesperance T, Crum J, Martone M, Volkmann N, Ellisman M H, and Weinberg R J (2012) Electron tomographic analysis of synaptic ultrastructure. J. Comp. Neurol. 520, 2697-2711.

Chen X, Winters C, and Reese R (2008) Life inside a thin section: tomography. J. Neurosci. 28, 9321-9327.

Fernández-Busnadiego R, Asano S, Oprisoreanu A M, Sakata E, Doengi M, Kochovski Z, Zürner M, Stein V, Schoch S, Baumeister W, and Lučić V (2013) Cryo-electron tomography reveals a critical role of RIM1 $\alpha$ in synaptic vesicle tethering. J. Cell Biol. 201, 725-740.

Fernández-Busnadiego R, Schrod N, Kochovski Z, Asano S, Vanhecke D, Baumeister W, and Lučić V (2011) Insights into the molecular organization of the neuron by cryo-electron tomography. J. Electron Microsc. (Tokyo) 60 Suppl 1, S137-S148.

Fernández-Busnadiego R, Zuber B, Maurer U, Cyrklaff M, Baumeister W, and Lučić V (2010) Quantitative analysis of the native presynaptic cytomatrix by cryoelectron tomography. J. Cell Biol. 188, 145-156.

Frey T G, Perkins G A, and Ellisman M H (2006) Electron tomography of membrane-bound cellular organelles. Annu. Rev. Biophys. Biomol. Struct. 35, 199-224.

Jou H T, Lee S, and Kim H J (2013) Improvement of alignment accuracy in electron tomography. Appl. Microsc. 43, 1-8.

Kim H W, Oh S H, Kim N, Nakazawa E, and Rhyu I J (2013) Rapid method for electron tomographic reconstruction and three-dimensional modeling of the murine synapse using an automated fiducial markerfree system. Microsc. Microanal. 19 Suppl 5, 182-187.

Kim J W, Lee S J, and Rhyu I J (2007) Construction of anaglyphic stereo pair image using Adobe Photoshop program. Korean J. Microsc. 37, 143-146.

Landis D, Hall A, Weinstein L, and Reese T (1988) The organization of cytoplasm at the presynaptic active zone of a central nervous system synapse. Neuron 1, 201-209.

Lee K J, Kim H, Kim T S, Park S H, and Rhyu I J (2004) Morphological analysis of spine shapes of Purkinje cell dendrites in the rat cerebellum using high-voltage electron microscopy. Neurosci. Lett. 359, 21-24.

Lučić V, Kossel A H, Yang T, Bonhoeffer T, Baumeister W, and Sartori A (2007) Multiscale imaging of neurons grown in culture: from light microscopy to cryo-electron tomography. J. Struct. Biol. 160, 146156.

Lučić V, Yang T, Schweikert G, Förster F, and Baumeister W (2005) Morphological characterization of molecular complexes present in the synaptic cleft. Structure 13, 423-434.

Mclntosh R, Mclntosh R, Nicastro D, and Mastronarde D (2005) New views of cells in 3D: an introduction to electron tomography. Trends Cell Biol. 15, 43-51.

Mun J Y, Lee K E, and Han S S (2008) Techniques for cryo-electron tomography in biological field. Korean J. Microsc. 38, 73-79.

Palade G and Palay S L (1954) Electron microscope observations of interneuronal and neuromuscular synapses. Anat. Rec. 118, 335336.

Rhyu I J and Park S N (2008) A glance of electron tomography through 4th international congress on electron tomogrpaphy. Korean J. Microsc. 38, 275-278.

Scout M C, Chen C C, Mecklenburg M, Zhu C, Xu R, Ercius P, Dahmen U, Regan B C, and Miao J (2012) Electron tomography at 2.4-ångström resolution. Nature 483, 444-447.

Siksou L, Rostaing P, Lechaire J P, Boudier T, Ohtsuka T, Fejtov A, Kao H T, Greengard P, Gundelfinger E, Triller A, and Marty S (2007) Threedimensional architecture of presynaptic terminal cytomatrix. J. Neurosci. 27, 6868-6877.

Siksou L, Varoqueaux F, Pascual O, Triller A, Brose N, and Marty S (2009) A common molecular basis for membrane docking and functional priming of synaptic vesicles. Eur. J. Neurosci. 30, 49-56. 